ESAIM: M2AN

Vol. 40, No 2, 2006, pp. 295-310

DOI: $10.1051 / \mathrm{m} 2 \mathrm{an}: 2006019$
ESAIM: Mathematical Modelling and Numerical Analysis

www.edpsciences.org/m2an

\title{
DYNAMIC FRICTIONAL CONTACT OF A VISCOELASTIC BEAM*
}

\author{
Marco Campo ${ }^{1}$, José R. Fernández ${ }^{1}$, Georgios E. Stavroulakis ${ }^{2}$ \\ AND JUAN M. VIAÑO ${ }^{1}$
}

\begin{abstract}
In this paper, we study the dynamic frictional contact of a viscoelastic beam with a deformable obstacle. The beam is assumed to be situated horizontally and to move, in both horizontal and tangential directions, by the effect of applied forces. The left end of the beam is clamped and the right one is free. Its horizontal displacement is constrained because of the presence of a deformable obstacle, the so-called foundation, which is modelled by a normal compliance contact condition. The effect of the friction is included in the vertical motion of the free end, by using Tresca's law or Coulomb's law. In both cases, the variational formulation leads to a nonlinear variational equation for the horizontal displacement coupled with a nonlinear variational inequality for the vertical displacement. We recall an existence and uniqueness result. Then, by using the finite element method to approximate the spatial variable and an Euler scheme to discretize the time derivatives, a numerical scheme is proposed. Error estimates on the approximative solutions are derived. Numerical results demonstrate the application of the proposed algorithm.
\end{abstract}

Mathematics Subject Classification. 65N15, 65N30, 74D05, 74M10, 74M15, 74S05, 74S20.

Received: September 28, 2005.

\section{INTRODUCTION}

Contact problems involving viscoplastic or viscoelastic materials appear in many industrial problems and everyday life (see, e.g., the monographs[18, 19,24] and references therein). Recently, one-dimensional contact problems for beams and rods have been studied[1,3,12,17,20,21], including the adhesion[15], the wear[11,22] or the damage[4]. Moreover, in[2] the adhesive contact problem of a membrane was studied.

In this work, we describe a model for the dynamic frictional contact of a viscoelastic beam with a deformable obstacle, the so-called foundation. The model was introduced in[1], where the existence of a unique weak solution was proved and its regularity was studied. That work was mainly focused on the mathematical aspects provided by the elastic case, that is, when the damping coefficient associated to the viscous effect is neglected.

\footnotetext{
Keywords and phrases. Dynamic unilateral contact, friction, viscoelastic beam, error estimates, numerical simulations.

* This work was partially supported by MCYT-Spain (Project BFM2003-05357). It is also part of the project "New Materials, Adaptive systems and their Nonlinearities; Modelling, Control and Numerical Simulation" carried out in the framework of the European Community program "Improving the Human Research Potential and the Socio-Economic Knowledge Base" (Contract No HPRN-CT-2002-00284)

${ }^{1}$ Departamento de Matemática Aplicada, Facultade de Matemáticas, Campus Sur s/n, Universidade de Santiago de Compostela, 15782 Santiago de Compostela, Spain. macampo@usc.es; jramon@usc.es; maviano@usc.es

2 Department of Production Engineering and Management, Technical University of Crete, 73100 Chania, Greece.

gestavr@dpem.tuc.gr
}

(c) EDP Sciences, SMAI 2006 


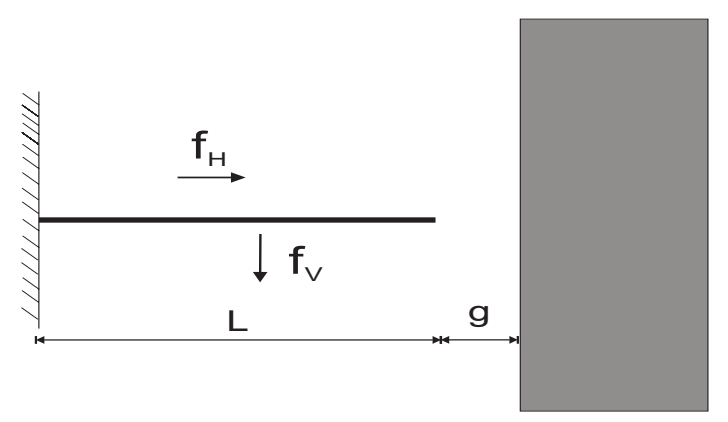

Figure 1. A viscoelastic beam in frictional contact.

Here, our aim is to study a numerical scheme for the viscoelastic case, based on the finite element method to approximate the spatial variable and an Euler scheme to discretize the time derivatives. Moreover, in order to show the accuracy of the proposed algorithm, some numerical simulations are presented.

\section{The MOdel AND VARIATIONAL FORMUlation}

In this section, we follow[1] and we describe the model for the dynamic evolution of a viscoelastic beam in frictional contact with a deformable obstacle. The beam is assumed to be rigidly clamped at its left end, and its right end is free to come into frictional contact with an obstacle situated at a distance $g$ to the right (see Fig. 1).

Let $[0, L], L>0$, be the reference configuration of the beam and let us denote by $[0, T], T>0$, the time interval of interest. The horizontal and vertical displacements of the beam are represented by $u(x, t)$ and $\tilde{u}(x, t)$, $(x, t) \in(0, L) \times(0, T)$, respectively.

The material is assumed viscoelastic and satisfying the Kelvin-Voigt viscoelastic laws[10],

$$
\begin{aligned}
& \sigma_{H}(x, t)=a_{1} u_{x}(x, t)+c_{1} u_{x t}(x, t), \quad(x, t) \in(0, L) \times(0, T), \\
& \sigma_{V}(x, t)=-\left(a_{2} \tilde{u}_{x x x}(x, t)+c_{2} \tilde{u}_{x x x t}(x, t)\right), \quad(x, t) \in(0, L) \times(0, T),
\end{aligned}
$$

where, as usual, a subscript denotes a partial derivative with respect to the corresponding variable. Here, $a_{1}, a_{2}>0$ are elastic constants, and $c_{1}, c_{2}>0$ represent the viscosities. We notice that these equations are decoupled because of the assumptions on the symmetry of the cross-section of the beam and the isotropy of its material.

Since we assume that the inertia effect is not negligible, the equations of motion have the following form,

$$
\begin{array}{ll}
\rho u_{t t}(x, t)-\left(\sigma_{H}\right)_{x}(x, t)=f_{H}(x, t), & (x, t) \in(0, L) \times(0, T), \\
\rho \tilde{u}_{t t}(x, t)-\left(\sigma_{V}\right)_{x}(x, t)=f_{V}(x, t), & (x, t) \in(0, L) \times(0, T),
\end{array}
$$

where $f_{H}$ and $f_{V}$ denote the horizontal and vertical applied forces, respectively. For simplicity, the density $\rho$ is assumed to be equal to 1 and

$$
f_{H} \in W^{1,2}\left(0, T ; L^{2}(0, L)\right), \quad f_{V} \in \mathcal{C}\left([0, T] ; L^{2}(0, L)\right) .
$$

Let $u_{0}, v_{0}, \tilde{u}_{0}, \tilde{v}_{0}$ be the initial conditions, that is,

$$
\begin{array}{lll}
u(x, 0)=u_{0}(x), & u_{t}(x, 0)=v_{0}(x), & x \in(0, L), \\
\tilde{u}(x, 0)=\tilde{u}_{0}(x), & \tilde{u}_{t}(x, 0)=\tilde{v}_{0}(x), & x \in(0, L) .
\end{array}
$$


To complete the model, we need to prescribe the boundary conditions. First, since the left end of the beam was supposed rigidly attached, we have

$$
\begin{aligned}
& u(0, t)=0, \quad t \in[0, T], \\
& \tilde{u}(0, t)=\tilde{u}_{x}(0, t)=0, \quad t \in[0, T] .
\end{aligned}
$$

Secondly, the beam is assumed to be located at a distance $g$ from the deformable obstacle. According to[16], the following normal compliance contact condition is considered,

$$
-\sigma_{H}(L, t)=c_{H}(u(L, t)-g)_{+}, \quad t \in[0, T],
$$

where $(u(L, t)-g)_{+}=\max \{0, u(L, t)-g\}$ and $c_{H}>0$ is a deformability coefficient (i.e. a hard spring or a penalty factor which is used to enforce the nonpenetration unilateral constraint).

Finally, concerning the vertical displacements, we assume that the sum of the moments on the free end is zero, i.e.

$$
a_{2} \tilde{u}_{x x}(L, t)+c_{2} \tilde{u}_{x x t}(L, t)=0, \quad t \in[0, T],
$$

and also a friction condition of the form, for a.e. $t \in[0, T]$,

$$
\left.\begin{array}{l}
\left|\sigma_{V}(L, t)\right| \leq h(t), \\
\text { if }\left|\sigma_{V}(L, t)\right|=h(t) \Rightarrow \exists \lambda \geq 0 ; \tilde{u}_{t}(L, t)=-\lambda \sigma_{V}(L, t), \\
\text { if }\left|\sigma_{V}(L, t)\right|<h(t) \Rightarrow \tilde{u}_{t}(L, t)=0 .
\end{array}\right\}
$$

In (12), function $h(t)$ represents a friction bound. We will consider two different cases:

(i) $h(t)=c_{V}=$ constant: it corresponds to the classical Tresca's conditions.

(ii) $h(t)=c_{V}(u(L, t)-g)_{+}$, for $c_{V}=$ constant $>0$ : it leads to a particular case of Coulomb's conditions.

Remark 1.1. We notice that the case (i) results from the case (ii), when the penetration $u(L, t)-g$ is assumed to be constant. Therefore, only the case (ii) will be considered in the rest of the paper.

The dynamic frictional contact problem of a viscoelastic beam with a deformable obstacle is written as follows.

Find a horizontal displacement $u:[0, L] \times[0, T] \rightarrow \mathbb{R}$ and a vertical displacement $\tilde{u}:[0, L] \times[0, T] \rightarrow \mathbb{R}$ which satisfy (1)-(12).

In order to derive a weak formulation of the above problem, we define the following variational spaces:

$$
\begin{aligned}
& H=L^{2}(0, L) \\
& E=\left\{w \in H^{1}(0, L) ; w(0)=0\right\} \\
& V=\left\{z \in H^{2}(0, L) ; z(0)=z_{x}(0)=0\right\} .
\end{aligned}
$$

Moreover, we denote by $(\cdot, \cdot)$ the classical inner product defined in $L^{2}(0, L)$ and, for a Hilbert space $X$, let $|\cdot|_{X}$ represent its norm. If $X^{\prime}$ denotes the dual space of $X$, then let $\langle\cdot, \cdot\rangle_{X^{\prime} X}$ be the duality pairing between $X^{\prime}$ and $X$.

Let $j_{H}(u, \cdot): E \rightarrow \mathbb{R}$ and $j_{V}(u, \cdot): V \rightarrow \mathbb{R}$ be the normal compliance and friction forms defined by

$$
\begin{array}{ll}
j_{H}(u, w)=c_{H}(u(L, t)-g)_{+} w(L, t), & \forall w \in E \\
j_{V}(u, z)=c_{V}(u(L, t)-g)_{+}|z(L, t)|, & \forall z \in V .
\end{array}
$$

Integrating by parts the equations of motion (3)-(4) and using the previously given boundary conditions, the variational formulation is then written as follows. 
Variational problem VP. Find the horizontal displacement $u:[0, T] \rightarrow E$ and the vertical displacement $\tilde{u}:[0, T] \rightarrow V$ such that $u(0)=u_{0}, u_{t}(0)=v_{0}, \tilde{u}(0)=\tilde{u}_{0}, \tilde{u}_{t}(0)=\tilde{v}_{0}$ and for a.e. $t \in(0, T)$,

$$
\left\langle u_{t t}(t), w\right\rangle_{E^{\prime} E}+a_{1}\left(u_{x}(t), w_{x}\right)+c_{1}\left(u_{x t}(t), w_{x}\right)+j_{H}(u(t), w)=\left(f_{H}(t), w\right), \quad \forall w \in E,
$$

$$
\begin{aligned}
\left\langle\tilde{u}_{t t}(t),\left(z-\tilde{u}_{t}(t)\right)\right\rangle_{V^{\prime} V}+\left(a_{2} \tilde{u}_{x x}(t)+c_{2} \tilde{u}_{x x t}(t),\left(z-\tilde{u}_{t}(t)\right)_{x x}\right) & \\
& +j_{V}(u(t), z)-j_{V}\left(u(t), \tilde{u}_{t}(t)\right) \geq\left(f_{V}(t),\left(z-\tilde{u}_{t}(t)\right)\right), \quad \forall z \in V .
\end{aligned}
$$

The existence of a unique solution to problem VP was proved in[1]. We recall the main result in the following.

Theorem 1.2. Let the assumption (5) hold and assume that $u_{0} \in E, \tilde{u}_{0} \in V$ and $v_{0}, \tilde{v}_{0} \in H$. Then, there exists a unique solution $\{u, \tilde{u}\}$ to problem VP with $u \in W^{1,2}(0, T ; E), u_{t t} \in L^{2}\left(0, T ; E^{\prime}\right), \tilde{u} \in W^{1,2}(0, T ; V)$ and $\tilde{u}_{t t} \in L^{2}\left(0, T ; V^{\prime}\right)$.

\section{Numerical approximation}

In this section we describe a fully discrete scheme for the variational problem VP. We discretize it in two steps, both the spatial and time variables. We introduce a uniform partition of the time interval with the step size $k=T / N$ and the nodes $t_{n}=n k, n=1, \ldots, N$. To discretize the spatial variable, we consider a uniform partition of $[0, L]$, denoted by $\left\{I_{i}\right\}_{i=1}^{M}$, in such a way that $[0, L]=\cup_{i=1}^{M} I_{i}$. We let $h$ denote the size of the partition. Let $V^{h}$ and $E^{h}$ be the following finite element spaces approximating $V$ and $E$,

$$
\begin{aligned}
& E^{h}=\left\{w^{h} \in E ; w_{\left.\right|_{I_{i}}}^{h} \in P_{1}\left(I_{i}\right), \quad 1 \leq i \leq M\right\}, \\
& V^{h}=\left\{z^{h} \in V ; z_{\left.\right|_{I_{i}}}^{h} \in P_{3}\left(I_{i}\right), \quad 1 \leq i \leq M\right\},
\end{aligned}
$$

where $P_{q}\left(I_{i}\right)$ denotes the polynomial space of degree less or equal to $q$ restricted to $I_{i}$. We note that, since $z^{h} \in V \subset H^{2}(0, L), z^{h} \in \mathcal{C}^{1}([0, L])$ and then $V^{h}$ is composed of $\mathcal{C}^{1}$ piecewise cubic functions, while $E^{h}$ is made of continuous piecewise affine functions.

We use the notation $z_{n}=z\left(t_{n}\right)$ for a continuous function $z(t)$, and for a sequence $\left\{z_{n}\right\}_{n=0}^{N}$ we denote by $\delta z_{n}=\left(z_{n}-z_{n-1}\right) / k$ the divided differences. Moreover, in this section no summation is assumed over a repeated index and $c$ denotes a generic constant which does not depend on $k, h$ or $n$.

For convenience, we will consider our variational problem in terms of the velocity fields $v(t)=\dot{u}(t), \tilde{v}(t)=\dot{\tilde{u}}(t)$ given by

$$
u(t)=\int_{0}^{t} v(s) \mathrm{d} s+u_{0}, \quad \tilde{u}(t)=\int_{0}^{t} \tilde{v}(s) \mathrm{d} s+\tilde{u}_{0} .
$$

Then, using an Euler scheme to discretize the time derivatives, we introduce the following fully discrete approximation of problem VP.

Fully discrete problem VP $\mathbf{V P}^{h k}$. Find $v^{h k}=\left\{v_{n}^{h k}\right\}_{n=0}^{N} \subset E^{h}$ and $\tilde{v}^{h k}=\left\{\tilde{v}_{n}^{h k}\right\}_{n=0}^{N} \subset V^{h}$, such that $u_{0}^{h k}=u_{0}^{h}$, $v_{0}^{h k}=v_{0}^{h}, \tilde{u}_{0}^{h k}=\tilde{u}_{0}^{h}, \tilde{v}_{0}^{h k}=\tilde{v}_{0}^{h}$ and, for $n=1, \ldots, N$,

$$
\left(\delta v_{n}^{h k}, w^{h}\right)+a_{1}\left(\left(u_{n}^{h k}\right)_{x}, w_{x}^{h}\right)+c_{1}\left(\left(v_{n}^{h k}\right)_{x}, w_{x}^{h}\right)+j_{H}\left(u_{n-1}^{h k}, w^{h}\right)=\left(\left(f_{H}\right)_{n}, w^{h}\right), \quad \forall w^{h} \in E^{h},
$$

$$
\begin{aligned}
\left(\delta \tilde{v}_{n}^{h k}, z^{h}-\tilde{v}_{n}^{h k}\right)+a_{2}\left(\left(\tilde{u}_{n}^{h k}\right)_{x x},\left(z^{h}-\tilde{v}_{n}^{h k}\right)_{x x}\right) & +c_{2}\left(\left(\tilde{v}_{n}^{h k}\right)_{x x},\left(z^{h}-\tilde{v}_{n}^{h k}\right)_{x x}\right) \\
& +j_{V}\left(u_{n}^{h k}, z^{h}\right)-j_{V}\left(u_{n}^{h k}, \tilde{v}_{n}^{h k}\right) \geq\left(\left(f_{V}\right)_{n}, z^{h}-\tilde{v}_{n}^{h k}\right), \quad \forall z^{h} \in V^{h}
\end{aligned}
$$

where $u_{0}^{h}, v_{0}^{h}, \tilde{u}_{0}^{h}$ and $\tilde{v}_{0}^{h}$ are appropriate approximations of the initial conditions $u_{0}, v_{0}$, $\tilde{u}_{0}$ and $\tilde{v}_{0}$, respectively. Moreover, $u^{h k}=\left\{u_{n}^{h k}\right\}_{n=0}^{N}$ and $\tilde{u}^{h k}=\left\{\tilde{u}_{n}^{h k}\right\}_{n=0}^{N}$ denote the displacement fields defined by

$$
u_{n}^{h k}=u_{n-1}^{h k}+k v_{n}^{h k}, \quad \tilde{u}_{n}^{h k}=\tilde{u}_{n-1}^{h k}+k \tilde{v}_{n}^{h k}, \quad n=1, \ldots, N .
$$


By induction, using classical results on variational inequalities (see[13]), we obtain that problem $\mathrm{VP}^{h k}$ admits a unique solution.

Our interest lies in estimating the errors $u_{n}-u_{n}^{h k}$ and $\tilde{u}_{n}-\tilde{u}_{n}^{h k}$. To that end, we make the following assumptions on the regularity of the solution,

$$
\begin{aligned}
& u \in \mathcal{C}^{1}([0, T] ; E), \quad \ddot{u} \in \mathcal{C}([0, T] ; H), \\
& \tilde{u} \in \mathcal{C}^{1}([0, T] ; V), \quad \tilde{\tilde{u}} \in \mathcal{C}([0, T] ; H) .
\end{aligned}
$$

The numerical analysis corresponding to the horizontal problem was done in [4], where the damage of the material and the thermal effects were also taken into account. Using the notation

$$
I_{j}=\left|\int_{0}^{t_{j}} v(s) \mathrm{d} s-\sum_{l=1}^{j} k v_{l}\right|_{E},
$$

the following error estimate was obtained.

Theorem 2.1. Let the assumptions of Theorem 1.2 hold. Under the regularity condition (18), the following error estimates are obtained for $n=0, \ldots, N$,

$$
\begin{array}{r}
\left|v_{n}-v_{n}^{h k}\right|_{H}^{2}+\sum_{j=1}^{n} k\left|v_{j}-v_{j}^{h k}\right|_{E}^{2} \leq c \sum_{j=1}^{n} k\left(\left|\dot{v}_{j}-\delta v_{j}\right|_{H}^{2}+I_{j}^{2}+\sum_{l=1}^{j-1} k\left|v_{l}-v_{l}^{h k}\right|_{E}^{2}+\left|u_{j}-u_{j-1}\right|_{E}^{2}+\left|v_{j}-w_{j}^{h}\right|_{E}^{2}\right) \\
+c\left(\left|u_{1}-u_{1}^{h}\right|_{H}^{2}+\left|u_{0}-u_{0}^{h}\right|_{E}^{2}+\left|v_{1}-w_{1}^{h}\right|_{H}^{2}+\left|v_{n}-w_{n}^{h}\right|_{H}^{2}\right)+c k^{-1} \sum_{j=1}^{n-1}\left|v_{j}-w_{j}^{h}-\left(v_{j+1}-w_{j+1}^{h}\right)\right|_{H}^{2}, \\
\forall\left\{w_{j}^{h}\right\}_{j=0}^{n} \subset V^{h} .
\end{array}
$$

Next, we let $z=\tilde{v}_{n}^{h k}$ in (14) at time $t=t_{n}$ to obtain, after a simple rearrangement,

$$
\begin{array}{r}
\left(\dot{\tilde{v}}_{n}, \tilde{v}_{n}-\tilde{v}_{n}^{h k}\right)+c_{2}\left(\left(\tilde{v}_{n}\right)_{x x},\left(\tilde{v}_{n}-\tilde{v}_{n}^{h k}\right)_{x x}\right)+a_{2}\left(\left(\tilde{u}_{n}\right)_{x x},\left(\tilde{v}_{n}-\tilde{v}_{n}^{h k}\right)_{x x}\right) \leq \\
j_{V}\left(u_{n}, \tilde{v}_{n}^{h k}\right)-j_{V}\left(u_{n}, \tilde{v}_{n}\right)+\left(\left(f_{V}\right)_{n}, \tilde{v}_{n}-\tilde{v}_{n}^{h k}\right),
\end{array}
$$

and the discrete variational inequality (17) is rewritten as follows,

$$
\begin{aligned}
-\left(\delta \tilde{v}_{n}^{h k}, \tilde{v}_{n}-\tilde{v}_{n}^{h k}\right)-c_{2}\left(\left(\tilde{v}_{n}^{h k}\right)_{x x},\left(\tilde{v}_{n}-\tilde{v}_{n}^{h k}\right)_{x x}\right)-a_{2}\left(\left(\tilde{u}_{n}^{h k}\right)_{x x},\left(\tilde{v}_{n}-\tilde{v}_{n}^{h k}\right)_{x x}\right) \leq \\
j_{V}\left(u_{n}^{h k}, z_{n}^{h}\right)-j_{V}\left(u_{n}^{h k}, \tilde{v}_{n}^{h k}\right)-\left(\left(f_{V}\right)_{n}, z_{n}^{h}-\tilde{v}_{n}^{h k}\right)-\left(\delta \tilde{v}_{n}^{h k}, \tilde{v}_{n}-z_{n}^{h}\right) \\
\quad-c_{2}\left(\left(\tilde{v}_{n}^{h k}\right)_{x x},\left(\tilde{v}_{n}-z_{n}^{h}\right)_{x x}\right)-a_{2}\left(\left(\tilde{u}_{n}^{h k}\right)_{x x},\left(\tilde{v}_{n}-z_{n}^{h}\right)_{x x}\right),
\end{aligned}
$$

for all $z_{n}^{h} \in V^{h}$. Adding these two inequalities, we obtain, for all $z_{n}^{h} \in V^{h}$,

$$
\begin{aligned}
\left(\dot{\tilde{v}}_{n}-\delta \tilde{v}_{n}^{h k}, \tilde{v}_{n}-\tilde{v}_{n}^{h k}\right)+c_{2}\left(\left(\tilde{v}_{n}-\tilde{v}_{n}^{h k}\right)_{x x},\left(\tilde{v}_{n}-\tilde{v}_{n}^{h k}\right)_{x x}\right)+a_{2}\left(\left(\tilde{u}_{n}-\tilde{u}_{n}^{h k}\right)_{x x},\left(\tilde{v}_{n}-\tilde{v}_{n}^{h k}\right)_{x x}\right) \leq \\
j_{V}\left(u_{n}, \tilde{v}_{n}^{h k}\right)-j_{V}\left(u_{n}, \tilde{v}_{n}\right)+j_{V}\left(u_{n}^{h k}, z_{n}^{h}\right)-j_{V}\left(u_{n}^{h k}, \tilde{v}_{n}^{h k}\right)-\left(\delta \tilde{v}_{n}^{h k}, \tilde{v}_{n}-z_{n}^{h}\right)+\left(\left(f_{V}\right)_{n}, \tilde{v}_{n}-z_{n}^{h}\right) \\
\quad-c_{2}\left(\left(\tilde{v}_{n}^{h k}\right)_{x x},\left(\tilde{v}_{n}-z_{n}^{h}\right)_{x x}\right)-a_{2}\left(\left(\tilde{u}_{n}^{h k}\right)_{x x},\left(\tilde{v}_{n}-z_{n}^{h}\right)_{x x}\right) .
\end{aligned}
$$

Now, let us define

$$
\begin{aligned}
R\left(u_{n}, \tilde{u}_{n}, z_{n}^{h}\right)=\left(\dot{\tilde{v}}_{n}, z_{n}^{h}-\tilde{v}_{n}\right)+c_{2}\left(\left(\tilde{v}_{n}\right)_{x x},\left(z_{n}^{h}-\tilde{v}_{n}\right)_{x x}\right) & \\
& +a_{2}\left(\left(\tilde{u}_{n}\right)_{x x},\left(z_{n}^{h}-\tilde{v}_{n}\right)_{x x}\right)+j_{V}\left(u_{n}, z_{n}^{h}\right)-j_{V}\left(u_{n}, \tilde{v}_{n}\right)-\left(\left(f_{V}\right)_{n}, z_{n}^{h}-\tilde{v}_{n}\right) .
\end{aligned}
$$


Writing $\dot{\tilde{v}}_{n}=\delta \tilde{v}_{n}-\left(\delta \tilde{v}_{n}-\dot{\tilde{v}}_{n}\right)$, after easy manipulations we get

$$
\begin{aligned}
\left(\delta \tilde{v}_{n}-\right. & \left.\delta \tilde{v}_{n}^{h k}, \tilde{v}_{n}-\tilde{v}_{n}^{h k}\right)+c_{2}\left(\left(\tilde{v}_{n}-\tilde{v}_{n}^{h k}\right)_{x x},\left(\tilde{v}_{n}-\tilde{v}_{n}^{h k}\right)_{x x}\right) \\
& \leq j_{V}\left(u_{n}, \tilde{v}_{n}^{h k}\right)-j_{V}\left(u_{n}, z_{n}^{h}\right)+j_{V}\left(u_{n}^{h k}, z_{n}^{h}\right)-j_{V}\left(u_{n}^{h k}, \tilde{v}_{n}^{h k}\right) \\
& +c_{2}\left(\left(\tilde{v}_{n}-\tilde{v}_{n}^{h k}\right)_{x x},\left(\tilde{v}_{n}-z_{n}^{h}\right)_{x x}\right)+a_{2}\left(\left(\tilde{u}_{n}-\tilde{u}_{n}^{h k}\right)_{x x},\left(\tilde{v}_{n}^{h k}-z_{n}^{h}\right)_{x x}\right) \\
& +\left(\tilde{\tilde{v}}_{n}-\delta \tilde{v}_{n}, \tilde{v}_{n}^{h k}-z_{n}^{h}\right)+R\left(u_{n}, \tilde{u}_{n}, z_{n}^{h}\right)+\left(\delta \tilde{v}_{n}-\delta \tilde{v}_{n}^{h k}, \tilde{v}_{n}-z_{n}^{h}\right) .
\end{aligned}
$$

From the definition of $j_{V}$, it follows that

$$
j_{V}\left(u_{n}, \tilde{v}_{n}^{h k}\right)-j_{V}\left(u_{n}, z_{n}^{h}\right)+j_{V}\left(u_{n}^{h k}, z_{n}^{h}\right)-j_{V}\left(u_{n}^{h k}, \tilde{v}_{n}^{h k}\right) \leq c\left|u_{n}-u_{n}^{h k}\right|_{E}\left(\left|\tilde{v}_{n}-\tilde{v}_{n}^{h k}\right|_{V}+\left|\tilde{v}_{n}-z_{n}^{h}\right|_{V}\right) .
$$

Since

$$
\left(\delta \tilde{v}_{n}-\delta \tilde{v}_{n}^{h k}, \tilde{v}_{n}-\tilde{v}_{n}^{h k}\right) \geq \frac{1}{2 k}\left[\left|\tilde{v}_{n}-\tilde{v}_{n}^{h k}\right|_{H}^{2}-\left|\tilde{v}_{n-1}-\tilde{v}_{n-1}^{h k}\right|_{H}^{2}\right]
$$

using the Cauchy's inequality $a b \leq \epsilon a^{2}+\frac{1}{4 \epsilon} b^{2}$ for $a, b, \epsilon>0$, the following estimate is obtained,

$$
\begin{aligned}
\left|\tilde{v}_{n}-\tilde{v}_{n}^{h k}\right|_{H}^{2}+c k\left|\tilde{v}_{n}-\tilde{v}_{n}^{h k}\right|_{V}^{2} \leq c k[\mid & u_{n}-\left.u_{n}^{h k}\right|_{E} ^{2}+\left|\dot{\tilde{v}}_{n}-\delta \tilde{v}_{n}\right|_{H}^{2}+\left|\tilde{v}_{n}-z_{n}^{h}\right|_{V}^{2} \\
& \left.\quad+\left|\tilde{u}_{n}-\tilde{u}_{n}^{h k}\right|_{V}^{2}+\left(\delta \tilde{v}_{n}-\delta \tilde{v}_{n}^{h k}, \tilde{v}_{n}-z_{n}^{h}\right)+\left|R\left(u_{n}, \tilde{u}_{n}, z_{n}^{h}\right)\right|\right]+\left|\tilde{v}_{n-1}-\tilde{v}_{n-1}^{h k}\right|_{H}^{2} .
\end{aligned}
$$

Then, an induction argument leads to

$$
\begin{aligned}
\left|\tilde{v}_{n}-\tilde{v}_{n}^{h k}\right|_{H}^{2}+k \sum_{j=1}^{n}\left|\tilde{v}_{j}-\tilde{v}_{j}^{h k}\right|_{V}^{2} \leq c k \sum_{j=1}^{n} & {\left[\left|u_{j}-u_{j}^{h k}\right|_{E}^{2}+\left|\dot{\tilde{v}}_{j}-\delta \tilde{v}_{j}\right|_{H}^{2}+\left|\tilde{v}_{j}-z_{j}^{h}\right|_{V}^{2}\right.} \\
& \left.+\left|\tilde{u}_{j}-\tilde{u}_{j}^{h k}\right|_{V}^{2}+\left(\delta \tilde{v}_{j}-\delta \tilde{v}_{j}^{h k}, \tilde{v}_{j}-z_{j}^{h}\right)+\left|R\left(u_{j}, \tilde{u}_{j}, z_{j}^{h}\right)\right|\right]+c\left|\tilde{v}_{0}-\tilde{v}_{0}^{h}\right|_{H}^{2} .
\end{aligned}
$$

Keeping in mind that

$\sum_{j=1}^{n} k\left(\delta \tilde{v}_{j}-\delta \tilde{v}_{j}^{h k}, \tilde{v}_{j}-z_{j}^{h}\right)=\left(\tilde{v}_{n}-\tilde{v}_{n}^{h k}, \tilde{v}_{n}-z_{n}^{h}\right)+\left(\tilde{v}_{0}^{h}-\tilde{v}_{0}, \tilde{v}_{1}-z_{1}^{h}\right)+\sum_{j=1}^{n-1}\left(\tilde{v}_{j}-\tilde{v}_{j}^{h k}, \tilde{v}_{j}-z_{j}^{h}-\left(\tilde{v}_{j+1}-z_{j+1}^{h}\right)\right)$,

and

$$
\begin{aligned}
\left|\tilde{u}_{j}-\tilde{u}_{j}^{h k}\right|_{V}^{2} \leq c\left(\left|\tilde{u}_{0}-\tilde{u}_{0}^{h}\right|_{V}^{2}+\tilde{I}_{j}^{2}+\sum_{l=1}^{j} k\left|\tilde{v}_{l}-\tilde{v}_{l}^{h k}\right|_{V}^{2}\right), \\
\left|u_{j}-u_{j}^{h k}\right|_{E}^{2} \leq c\left(\left|u_{0}-u_{0}^{h}\right|_{E}^{2}+I_{j}^{2}+\sum_{l=1}^{j} k\left|v_{l}-v_{l}^{h k}\right|_{E}^{2}\right),
\end{aligned}
$$


where $\tilde{I}_{j}=\left|\int_{0}^{t_{j}} \tilde{v}(s) \mathrm{d} s-\sum_{l=1}^{j} k \tilde{v}_{l}\right|_{V}$ is the integration error, the following estimate is obtained:

$$
\begin{aligned}
\left|\tilde{v}_{n}-\tilde{v}_{n}^{h k}\right|_{H}^{2}+ & k \sum_{j=1}^{n}\left|\tilde{v}_{j}-\tilde{v}_{j}^{h k}\right|_{V}^{2} \leq c k \sum_{j=1}^{n}\left[\sum_{k=1}^{l} k\left|v_{l}-v_{l}^{h k}\right|_{E}^{2}+I_{j}^{2}+\left|\dot{\tilde{v}}_{j}-\delta \tilde{v}_{j}\right|_{H}^{2}\right. \\
& \left.+\left|\tilde{v}_{j}-z_{j}^{h}\right|_{V}^{2}+\tilde{I}_{j}^{2}+\sum_{l=1}^{j} k\left|\tilde{v}_{l}-\tilde{v}_{l}^{h k}\right|_{V}^{2}+\left|R\left(u_{j}, \tilde{u}_{j}, z_{j}^{h}\right)\right|\right]+c\left|\tilde{u}_{0}-\tilde{u}_{0}^{h}\right|_{V}^{2}+c\left|\tilde{v}_{0}-\tilde{v}_{0}^{h}\right|_{H}^{2} \\
& +c\left|\tilde{v}_{1}-z_{1}^{h}\right|_{H}^{2}+c\left|\tilde{v}_{n}-z_{n}^{h}\right|_{H}^{2}+c\left|u_{0}-u_{0}^{h}\right|_{E}^{2}+c k^{-1} \sum_{j=1}^{n-1}\left|\tilde{v}_{j}-z_{j}^{h}-\left(\tilde{v}_{j+1}-z_{j+1}^{h}\right)\right|_{H}^{2}
\end{aligned}
$$

Adding now (20) and (23), we get

$$
\begin{aligned}
\mid v_{n}- & \left.v_{n}^{h k}\right|_{H} ^{2}+k \sum_{j=1}^{n}\left|v_{j}-v_{j}^{h k}\right|_{E}^{2}+\left|\tilde{v}_{n}-\tilde{v}_{n}^{h k}\right|_{H}^{2}+k \sum_{j=1}^{n}\left|\tilde{v}_{j}-\tilde{v}_{j}^{h k}\right|_{V}^{2} \\
\leq & c\left\{\sum _ { j = 1 } ^ { n } k \left[\left|\tilde{\tilde{v}}_{j}-\delta \tilde{v}_{j}\right|_{H}^{2}+\left|\dot{v}_{j}-\delta v_{j}\right|_{H}^{2}+\left|u_{j}-u_{j-1}\right|_{E}^{2}+\left|v_{j}-w_{j}^{h}\right|_{E}^{2}\right.\right. \\
& +\left|\tilde{v}_{j}-z_{j}^{h}\right|_{V}^{2}+\tilde{I}_{j}^{2}+I_{j}^{2}+\sum_{l=1}^{j} k\left|\tilde{v}_{l}-\tilde{v}_{l}^{h k}\right|_{V}^{2}+\sum_{l=1}^{j} k\left|v_{l}-v_{l}^{h k}\right|_{E}^{2} \\
& \left.+\left|R\left(u_{j}, \tilde{u}_{j}, z_{j}^{h}\right)\right|\right]+\left|v_{0}-v_{0}^{h}\right|_{H}^{2}+\left|\tilde{v}_{0}-\tilde{v}_{0}^{h}\right|_{H}^{2}+\left|u_{0}-u_{0}^{h}\right|_{E}^{2}+\left|\tilde{u}_{0}-\tilde{u}_{0}^{h}\right|_{V}^{2} \\
& +\left|v_{1}-w_{1}^{h}\right|_{H}^{2}+\left|\tilde{v}_{1}-z_{1}^{h}\right|_{H}^{2}+\left|v_{n}-w_{n}^{h}\right|_{H}^{2}+\left|\tilde{v}_{n}-z_{n}^{h}\right|_{H}^{2} \\
& \left.+k^{-1} \sum_{j=1}^{n-1}\left|v_{j}-w_{j}^{h}-\left(v_{j+1}-w_{j+1}^{h}\right)\right|_{H}^{2}+k^{-1} \sum_{j=1}^{n-1}\left|\tilde{v}_{j}-z_{j}^{h}-\left(\tilde{v}_{j+1}-z_{j+1}^{h}\right)\right|_{H}^{2}\right\}
\end{aligned}
$$

Let us define the following quantities for $n=1, \ldots, N$,

$$
\begin{aligned}
e_{n}= & \left|v_{n}-v_{n}^{h k}\right|_{H}^{2}+k \sum_{j=1}^{n}\left|v_{j}-v_{j}^{h k}\right|_{E}^{2}+\left|\tilde{v}_{n}-\tilde{v}_{n}^{h k}\right|_{H}^{2}+k \sum_{j=1}^{n}\left|\tilde{v}_{j}-\tilde{v}_{j}^{h k}\right|_{V}^{2}, \\
g_{n}= & \sum_{j=1}^{n} k\left[\left|\dot{\tilde{v}}_{j}-\delta \tilde{v}_{j}\right|_{H}^{2}+\left|\dot{v}_{j}-\delta v_{j}\right|_{H}^{2}+\left|u_{j}-u_{j-1}\right|_{E}^{2}+\left|v_{j}-w_{j}^{h}\right|_{E}^{2}\right. \\
& \left.+\left|\tilde{v}_{j}-z_{j}^{h}\right|_{V}^{2}+\tilde{I}_{j}^{2}+I_{j}^{2}+\left|R\left(u_{j}, \tilde{u}_{j}, z_{j}^{h}\right)\right|\right]+\left|v_{0}-v_{0}^{h}\right|_{H}^{2}+\left|\tilde{v}_{0}-\tilde{v}_{0}^{h}\right|_{H}^{2} \\
& +\left|u_{0}-u_{0}^{h}\right|_{E}^{2}+\left|\tilde{u}_{0}-\tilde{u}_{0}^{h}\right|_{V}^{2}+\left|v_{1}-w_{1}^{h}\right|_{H}^{2}+\left|\tilde{v}_{1}-z_{1}^{h}\right|_{H}^{2} \\
& +\left|v_{n}-w_{n}^{h}\right|_{H}^{2}+\left|\tilde{v}_{n}-z_{n}^{h}\right|_{H}^{2}+k^{-1} \sum_{j=1}^{n-1}\left|v_{j}-w_{j}^{h}-\left(v_{j+1}-w_{j+1}^{h}\right)\right|_{H}^{2} \\
& +k^{-1} \sum_{j=1}^{n-1}\left|\tilde{v}_{j}-z_{j}^{h}-\left(\tilde{v}_{j+1}-z_{j+1}^{h}\right)\right|_{H}^{2},
\end{aligned}
$$


and

$$
e_{0}=g_{0}=\left|v_{0}-v_{0}^{h}\right|_{H}^{2}+\left|\tilde{v}_{0}-\tilde{v}_{0}^{h}\right|_{H}^{2}
$$

Therefore, estimate (24) implies that

$$
e_{0} \leq g_{0} \quad \text { and } \quad e_{n} \leq c g_{n}+c \sum_{j=1}^{n} k_{j} e_{j}, \quad n=1, \ldots, N,
$$

where $k_{j}=k, j=1, \ldots, N$. Then, we use the following discrete version of the Gronwall's inequality (see[14]).

Lemma 2.2. Assume that $\left\{g_{n}\right\}_{n=0}^{N},\left\{e_{n}\right\}_{n=0}^{N}$ and $\left\{k_{n}\right\}_{n=1}^{N}$ are three sequences of nonnegative numbers satisfying

$$
\begin{aligned}
& e_{0} \leq g_{0}, \\
& e_{n} \leq c g_{n}+c \sum_{j=1}^{n} k_{j} e_{j}, \quad n=1, \ldots, N,
\end{aligned}
$$

where $c$ is a positive constant. Then, there exists a positive constant $d>0$ such that

$$
\max _{0 \leq n \leq N} e_{n} \leq d \max _{0 \leq n \leq N} g_{n}
$$

Applying Lemma 2.2, we obtain the following result.

Theorem 2.3. Let the assumptions of Theorem 1.2 hold true. Let us assume the additional regularity conditions (18)-(19). Then, the following error estimates are obtained for all $\left\{w_{j}^{h}\right\}_{j=0}^{N} \subset E^{h},\left\{z_{j}^{h}\right\}_{j=0}^{N} \subset V^{h}$,

$$
\begin{aligned}
\max _{0 \leq n \leq N} & \left\{\left|v_{n}-v_{n}^{h k}\right|_{H}^{2}+\left|\tilde{v}_{n}-\tilde{v}_{n}^{h k}\right|_{H}^{2}\right\}+k \sum_{j=1}^{N}\left[\left|v_{j}-v_{j}^{h k}\right|_{E}^{2}+\left|\tilde{v}_{j}-\tilde{v}_{j}^{h k}\right|_{V}^{2}\right] \\
\leq & c\left\{\sum _ { j = 1 } ^ { N } k \left[\left|\dot{v}_{j}-\delta v_{j}\right|_{H}^{2}+\left|\dot{\tilde{v}}_{j}-\delta \tilde{v}_{j}\right|_{H}^{2}+\left|u_{j}-u_{j-1}\right|_{E}^{2}+\left|v_{j}-w_{j}^{h}\right|_{E}^{2}\right.\right. \\
& \left.+\left|\tilde{v}_{j}-z_{j}^{h}\right|_{V}^{2}+\tilde{I}_{j}^{2}+I_{j}^{2}+\left|R\left(u_{j}, \tilde{u}_{j}, z_{j}^{h}\right)\right|\right]+\left|v_{0}-v_{0}^{h}\right|_{H}^{2}+\left|\tilde{v}_{0}-\tilde{v}_{0}^{h}\right|_{H}^{2} \\
& +k^{-1} \sum_{j=1}^{N-1}\left|v_{j}-w_{j}^{h}-\left(v_{j+1}-w_{j+1}^{h}\right)\right|_{H}^{2}+k^{-1} \sum_{j=1}^{N-1}\left|\tilde{v}_{j}-z_{j}^{h}-\left(\tilde{v}_{j+1}-z_{j+1}^{h}\right)\right|_{H}^{2} \\
& \left.+\left|u_{0}-u_{0}^{h}\right|_{E}^{2}+\left|\tilde{u}_{0}-\tilde{u}_{0}^{h}\right|_{V}^{2}+\max _{0 \leq n \leq N}\left|v_{n}-w_{n}^{h}\right|_{H}^{2}+\max _{0 \leq n \leq N}\left|\tilde{v}_{n}-z_{n}^{h}\right|_{H}^{2}\right\} .
\end{aligned}
$$

The inequality (25) is the basis for deriving the convergence rate. Let $\Pi^{h}$ and $\tilde{\Pi}^{h}$ denote the standard Lagrange and Hermite interpolation operators associated to the finite element spaces $E^{h}$ or $V^{h}$ (see[9]), respectively. Assume that the initial conditions $u_{0}, v_{0}, \tilde{u}_{0}$ and $\tilde{v}_{0}$ are such that

$$
u_{0} \in H^{2}(0, L), \quad v_{0} \in H^{1}(0, L), \quad \tilde{u}_{0} \in H^{3}(0, L), \quad \tilde{v}_{0} \in H^{1}(0, L),
$$

and define the discrete initial conditions by

$$
u_{0}^{h}=\Pi^{h} u_{0}, \quad v_{0}^{h}=\Pi^{h} v_{0}, \quad \tilde{u}_{0}^{h}=\tilde{\Pi}^{h} \tilde{u}_{0}, \quad \tilde{v}_{0}^{h}=\Pi^{h} \tilde{v}_{0} .
$$

Then, we have the first error estimate. 
Corollary 2.4. Let the assumptions of Theorem 1.2 hold true. Under the following additional regularity conditions

$$
\begin{array}{ll}
u \in H^{2}(0, T ; E) \cap \mathcal{C}^{1}\left([0, T] ; H^{2}(0, L)\right), & \dddot{u} \in L^{2}(0, T ; H), \\
\tilde{u} \in H^{2}(0, T ; V) \cap \mathcal{C}^{1}\left([0, T] ; H^{3}(0, L)\right), & \dddot{\tilde{u}} \in L^{2}(0, T ; H),
\end{array}
$$

we have the following error estimate,

$$
\max _{0 \leq n \leq N}\left\{\left|u_{n}-u_{n}^{h k}\right|_{H}+\left|\tilde{u}_{n}-\tilde{u}_{n}^{h k}\right|_{H}\right\} \leq c\left(h^{1 / 2}+k\right)
$$

Proof. We note that

$$
\begin{aligned}
& k \sum_{j=1}^{N}\left[\left|\dot{v}_{j}-\delta v_{j}^{h k}\right|_{H}^{2}+\left|\dot{\tilde{v}}_{j}-\delta \tilde{v}_{j}^{h k}\right|_{H}^{2}\right] \leq c k^{2}\left[|\dddot{u}|_{L^{2}(0, T ; H)}^{2}+|\dddot{\tilde{u}}|_{L^{2}(0, T ; H)}^{2}\right], \\
& k \sum_{j=1}^{N}\left|u_{j}-u_{j-1}\right|_{E}^{2} \leq c k^{2}|\dot{u}|_{L^{2}(0, T ; E)}^{2}, \\
& \max _{1 \leq n \leq N} I_{n} \leq c k|u|_{H^{2}(0, T ; E)}, \quad \max _{1 \leq n \leq N} \tilde{I}_{n} \leq c k|\tilde{u}|_{H^{2}(0, T ; V)} .
\end{aligned}
$$

We also have (see[7,9] for details),

$$
\begin{aligned}
& k^{-1} \sum_{j=1}^{N-1}\left|\left(v_{j+1}-w_{j+1}^{h}\right)-\left(v_{j}-w_{j}^{h}\right)\right|_{H}^{2} \leq c h^{2}|\dot{v}|_{L^{2}(0, T ; E)}^{2}, \\
& k^{-1} \sum_{j=1}^{N-1}\left|\left(\tilde{v}_{j+1}-z_{j+1}^{h}\right)-\left(\tilde{v}_{j}-z_{j}^{h}\right)\right|_{H}^{2} \leq c h^{2}|\dot{\tilde{v}}|_{L^{2}(0, T ; V)}^{2}, \\
& \left|R\left(u_{j}, \tilde{u}_{j}, z_{j}^{h}\right)\right| \leq c\left|\tilde{v}_{j}-z_{j}^{h}\right|_{V} \leq c h|\tilde{u}|_{C^{1}\left([0, T] ; H^{3}(0, L)\right)} .
\end{aligned}
$$

Using (21) and (22) it follows that

$$
\begin{aligned}
\max _{0 \leq n \leq N}\left\{\left|u_{n}-u_{n}^{h k}\right|_{E}^{2}+\left|\tilde{u}_{n}-\tilde{u}_{n}^{h k}\right|_{V}^{2}\right\} \leq c\left(\left|u_{0}-u_{0}^{h}\right|_{E}^{2}+\left|\tilde{u}_{0}-\tilde{u}_{0}^{h}\right|_{V}^{2}\right. & \\
& \left.+\max _{0 \leq n \leq N}\left\{I_{n}^{2}+\tilde{I}_{n}^{2}\right\}+\sum_{j=1}^{N} k\left[\left|v_{j}-v_{j}^{h k}\right|_{E}^{2}+\left|\tilde{v}_{j}-\tilde{v}_{j}^{h k}\right|_{V}^{2}\right]\right)
\end{aligned}
$$

and the rest of the terms in (25) are bounded using the approximation properties of the finite element spaces $E^{h}$ and $V^{h}$ (see[9]).

Remark 2.5. We notice that (26) is just an example of an estimate which is based on the previous regularity assumptions. If we assume further regularity assumptions on the continuous solution, the linear convergence of the algorithm is achieved. For instance, if $\tilde{u} \in \mathcal{C}^{1}\left([0, T] ; \mathcal{C}^{3}([0, L])\right)$ (which implies that $\sigma_{V} \in \mathcal{C}([0, T] ; \mathcal{C}([0, L]))$, we have

and then,

$$
\left|R\left(u_{j}, \tilde{u}_{j}, z_{j}^{h}\right)\right| \leq c\left|\tilde{v}_{j}-z_{j}^{h}\right|_{H^{1}(0, L)} \leq c h^{2}\left|\tilde{v}_{j}\right|_{H^{3}(0, L)},
$$

$$
\max _{0 \leq n \leq N}\left\{\left|u_{n}-u_{n}^{h k}\right|_{H}+\left|\tilde{u}_{n}-\tilde{u}_{n}^{h k}\right|_{H}\right\} \leq c(h+k) .
$$




\section{NumericAl RESUlts}

In order to show the behaviour of the numerical scheme presented in the above section, some numerical experiments have been done. In this section we describe the algorithm employed to solve the fully discrete problem $\mathrm{VP}^{h k}$, and we briefly present some numerical results which demonstrate the performance of the method.

\subsection{Numerical resolution}

First we have, for $n=1, \ldots, N$, that the horizontal velocity is the unique solution of the following variational equation,

$$
\begin{aligned}
&\left(\rho \delta v_{n}^{h k}, w^{h}\right)+a_{1} k\left(\left(v_{n}^{h k}\right)_{x}, w_{x}^{h}\right)+c_{1}\left(\left(v_{n}^{h k}\right)_{x}, w_{x}^{h}\right)+j_{H}\left(u_{n-1}^{h k}, w^{h}\right)= \\
&\left(\left(f_{H}\right)_{n}, w^{h}\right)-a_{1}\left(\left(u_{n-1}^{h k}\right)_{x}, w_{x}^{h}\right), \quad \forall w^{h} \in E^{h}
\end{aligned}
$$

Since problem (27) corresponds to a linear system, its resolution is done using Cholesky's method.

Secondly, the discrete vertical velocity is obtained from the solution of the following variational inequality,

$$
\begin{aligned}
\left(\rho \delta \tilde{v}_{n}^{h k}, z^{h}-\tilde{v}_{n}^{h k}\right)+a_{2}\left(\left(\tilde{u}_{n}^{h k}\right)_{x x},\left(z^{h}-\tilde{v}_{n}^{h k}\right)_{x x}\right)+c_{2}\left(\left(\tilde{v}_{n}^{h k}\right)_{x x},\left(z^{h}-\tilde{v}_{n}^{h k}\right)_{x x}\right) & \\
& +j_{V}\left(u_{n}^{h k}, z^{h}\right)-j_{V}\left(u_{n}^{h k}, \tilde{v}_{n}^{h k}\right) \geq\left(\left(f_{V}\right)_{n}, z^{h}-\tilde{v}_{n}^{h k}\right), \quad \forall z^{h} \in V^{h}
\end{aligned}
$$

We note that problem (28) is a second kind variational inequality with the difficulty provided by the nondifferentiability of the operator $j_{V}$. This can be solved using algorithms, for example, of Uzawa's type[8]. Nevertheless, we introduce here an efficient combination of a penalty-duality algorithm with a penalization over the friction condition. The penalized friction condition consists, for $0<\mu$, in the following

$$
-\sigma_{V}(L, t)=\Phi_{\mu}(\tilde{v}(L, t)), \text { with } \Phi_{\mu}(r)= \begin{cases}-h(t) & \text { if } \quad r<-\mu, \\ \frac{h(t)}{\mu} r & \text { if } \quad r \in[-\mu, \mu], \\ h(t) & \text { if } \quad r>\mu .\end{cases}
$$

For further smoothing techniques of the frictional problem the reader may see [23, 25].

Using (29) instead of (12), another second kind variational inequality is derived for the vertical velocity,

$$
\begin{aligned}
\left(\rho \delta \tilde{v}_{\mu}^{h k}, z^{h}-\tilde{v}_{\mu}^{h k}\right)+a_{2}\left(\left(\tilde{u}_{\mu}^{h k}\right)_{x x},\left(z^{h}-\tilde{v}_{\mu}^{h k}\right)_{x x}\right) & +c_{2}\left(\left(\tilde{v}_{\mu}^{h k}\right)_{x x},\left(z^{h}-\tilde{v}_{\mu}^{h k}\right)_{x x}\right) \\
& +j_{\mu}\left(u_{n}^{h k}, z^{h}\right)-j_{\mu}\left(u_{n}^{h k}, \tilde{v}_{\mu}^{h k}\right) \geq\left(\left(f_{V}\right)_{n}, z^{h}-\tilde{v}_{\mu}^{h k}\right), \quad \forall z^{h} \in V^{h}
\end{aligned}
$$

where $j_{\mu}\left(u_{n}^{h k}, \cdot\right): V \rightarrow \mathbb{R}$ is a differentiable functional defined by

$$
j_{\mu}\left(u_{n}^{h k}, v\right)= \begin{cases}-h\left(u_{n}^{h k}\right) v-h\left(u_{n}^{h k}\right) \frac{\mu}{2} & \text { if } \quad v<-\mu, \\ \frac{h\left(u_{n}^{h k}\right)}{2 \mu} v^{2} & \text { if } \quad v \in[-\mu, \mu], \\ h\left(u_{n}^{h k}\right) v-h\left(u_{n}^{h k}\right) \frac{\mu}{2} & \text { if } \quad v>\mu,\end{cases}
$$

where either $h\left(u_{n}^{h k}\right)=$ constant (Tresca's law) or $h\left(u_{n}^{h k}\right)=c_{V}\left(u_{n}^{h k}-g\right)_{+}$(Coulomb's law).

Problem (30) is solved using the penalty-duality algorithm introduced in[5]. Moreover, in[6] it was proved that

$$
\left|\tilde{v}_{n}^{h k}-\tilde{v}_{\mu}^{h k}\right|_{V} \leq c \mu\left(h+k+|\tilde{v}|_{\mathcal{C}([0, T] ; V)}\right)
$$


TABLE 1. Example 1: numerical errors for some $n$ and $k$ (stick case).

\begin{tabular}{ccccccc}
\hline$n \downarrow k \rightarrow$ & 0.01 & 0.005 & 0.002 & 0.001 & 0.0005 & 0.0001 \\
\hline 100 & $5.042 \mathrm{e}-3$ & $5.042 \mathrm{e}-3$ & $5.042 \mathrm{e}-3$ & $5.042 \mathrm{e}-3$ & $5.042 \mathrm{e}-3$ & $5.042 \mathrm{e}-3$ \\
200 & $2.856 \mathrm{e}-3$ & $2.856 \mathrm{e}-3$ & $2.856 \mathrm{e}-3$ & $2.856 \mathrm{e}-3$ & $2.856 \mathrm{e}-3$ & $2.856 \mathrm{e}-3$ \\
400 & $1.699 \mathrm{e}-3$ & $1.699 \mathrm{e}-3$ & $1.699 \mathrm{e}-3$ & $1.699 \mathrm{e}-3$ & $1.699 \mathrm{e}-3$ & $1.699 \mathrm{e}-3$ \\
800 & $1.008 \mathrm{e}-3$ & $1.008 \mathrm{e}-3$ & $1.008 \mathrm{e}-3$ & $1.008 \mathrm{e}-3$ & $1.008 \mathrm{e}-3$ & $1.008 \mathrm{e}-3$ \\
1600 & $5.639 \mathrm{e}-4$ & $5.637 \mathrm{e}-4$ & $5.637 \mathrm{e}-4$ & $5.636 \mathrm{e}-4$ & $5.636 \mathrm{e}-4$ & $5.636 \mathrm{e}-4$ \\
3200 & $2.028 \mathrm{e}-4$ & $2.015 \mathrm{e}-4$ & $2.008 \mathrm{e}-4$ & $2.007 \mathrm{e}-4$ & $2.005 \mathrm{e}-4$ & $2.003 \mathrm{e}-4$ \\
\hline
\end{tabular}
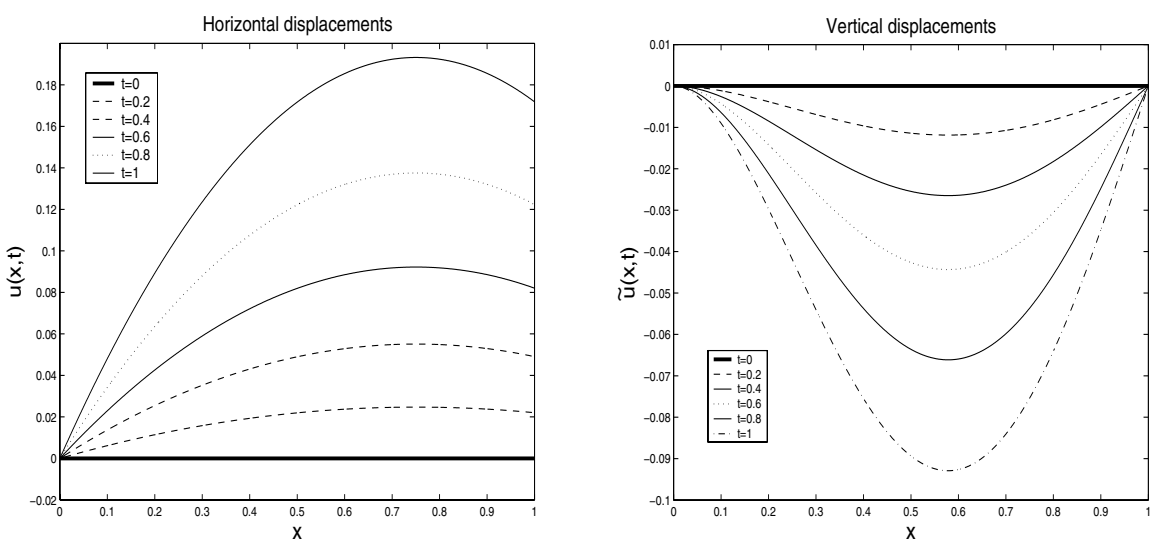

Figure 2. Example 1: horizontal and vertical displacements at different instants.

\subsection{Numerical simulations}

\subsubsection{First example: stick case}

As a first example, we have considered a beam of length $L=1 \mathrm{~m}$ with its left end rigidly attached, and no gap is assumed between the right end and the obstacle $(g=0 \mathrm{~m})$. Coulomb's contact conditions were employed.

The following data were used in the simulations:

$$
\begin{aligned}
& T=1 \mathrm{~s}, \quad a_{1}=a_{2}=1000 \mathrm{~N}, \quad c_{1}=c_{2}=1 \mathrm{~N} \cdot \mathrm{s}, \\
& \rho=10^{-4} \mathrm{~kg} / \mathrm{m}^{3}, \quad c_{H}=10^{3}, \quad c_{V}=5 \times 10^{6}, \quad \mu=10^{-10}, \\
& f_{H}(x, t)=400\left(e^{t}-1\right) \mathrm{N} / \mathrm{m}, \quad f_{V}(x, t)=-10000\left(e^{t}-1\right) \mathrm{N} / \mathrm{m} \text { in }[0,1], \\
& u_{0}(x)=\tilde{u}_{0}(x)=0 \mathrm{~m}, \quad v_{0}(x)=\tilde{v}_{0}(x)=0 \mathrm{~m} / \mathrm{s} \quad \text { in }[0,1] .
\end{aligned}
$$

In order to see the behaviour of the algorithm, a sequence of numerical solutions, based on uniform partitions of both the time interval and the spatial domain, have been computed. Here, the spatial domain $[0,1]$ is divided into $n$ equal parts $(h=1 / n)$. We start with $n=100$, which is successively halved, and $k=0.5$, taking as "exact" solution that obtained with $n=12800$ and $k=10^{-5}$. In Table 1 , the numerical errors

$$
\max _{o \leq n \leq N}\left\{\left|u_{n}-u_{n}^{h k}\right|_{H}+\left|\tilde{u}_{n}-\tilde{u}_{n}^{h k}\right|_{H}\right\}
$$

obtained with different discretization parameters, are shown. As we can see, the convergence of the numerical scheme is deduced. 


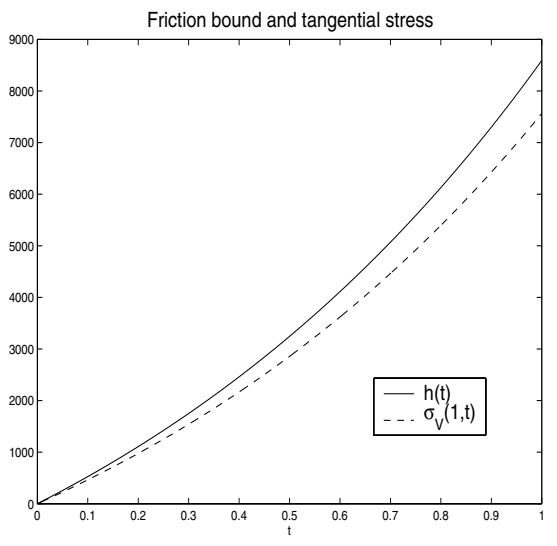

Figure 3. Example 1: evolution in time of the tangential stress $\sigma_{V}(1, t)$ and the friction bound $h(t)=c_{V} u(1, t)$.

TABLE 2. Example 2: numerical errors for some $n$ and $k$ (Slip case).

\begin{tabular}{ccccccc}
\hline$n \downarrow k \rightarrow$ & 0.01 & 0.005 & 0.002 & 0.001 & 0.0005 & 0.0001 \\
\hline 100 & $1.522 \mathrm{e}-2$ & $1.060 \mathrm{e}-2$ & $7.923 \mathrm{e}-3$ & $7.056 \mathrm{e}-3$ & $6.598 \mathrm{e}-3$ & $5.944 \mathrm{e}-3$ \\
200 & $1.512 \mathrm{e}-2$ & $1.044 \mathrm{e}-2$ & $7.627 \mathrm{e}-3$ & $6.669 \mathrm{e}-3$ & $6.156 \mathrm{e}-3$ & $5.417 \mathrm{e}-3$ \\
400 & $1.512 \mathrm{e}-2$ & $1.043 \mathrm{e}-2$ & $7.610 \mathrm{e}-3$ & $6.649 \mathrm{e}-3$ & $6.133 \mathrm{e}-3$ & $5.382 \mathrm{e}-3$ \\
800 & $1.514 \mathrm{e}-2$ & $1.045 \mathrm{e}-2$ & $7.634 \mathrm{e}-3$ & $6.674 \mathrm{e}-3$ & $6.159 \mathrm{e}-3$ & $5.409 \mathrm{e}-3$ \\
1600 & $1.516 \mathrm{e}-2$ & $1.049 \mathrm{e}-2$ & $7.670 \mathrm{e}-3$ & $6.718 \mathrm{e}-3$ & $6.200 \mathrm{e}-3$ & $5.453 \mathrm{e}-3$ \\
3200 & $1.498 \mathrm{e}-2$ & $1.044 \mathrm{e}-2$ & $7.702 \mathrm{e}-3$ & $6.755 \mathrm{e}-3$ & $6.281 \mathrm{e}-3$ & $5.549 \mathrm{e}-3$ \\
\hline
\end{tabular}

Using $h=k=10^{-3}$ as discretization parameters, in Figure 2 (left-hand side), the horizontal displacements are shown at different times, while the vertical displacements are depicted at the same times on the righthand side. We can observe that the right end of the beam keeps clamped, because the tangential stress does not achieve the friction bound, and the beam remains sticked to the obstacle (see Fig. 3).

\subsubsection{Second example: slip case}

As a second example, we consider the same setting that in the above example. However, we assume now that the horizontal force is given by $f_{H}(x, t)=100\left(e^{t}-1\right) N / m$ for $x \in[0,1]$, the vertical one by $f_{V}(x, t)=$ $-1000\left(e^{t}-1\right) N / m$ for $x \in[0,1]$ and the friction coefficient $c_{V}=10^{4}$. We notice that with these new data, there is always movement of the contact node since the tangential stress equals the friction bound.

We have computed a sequence of numerical solutions following the previous example. In Table 2 , the numerical errors (31) are depicted for different discretization parameters. Again, the convergence of the algorithm is shown.

Using $h=k=10^{-3}$, the vertical displacements at different times are shown on the left-hand side of Figure 4. On the right-hand side, the evolution in time of the vertical displacement of the contact node is plotted. As we can observe, the right end of the beam is always moving.

\subsubsection{Third example: a test with Tresca's conditions}

As a third example, we consider a viscoelastic beam of length $L=1 \mathrm{~m}$ which is rigidly attached at its left end. Tresca's conditions are employed for the modelling of the contact. Since the problem decouples and the horizontal displacement was considered in previous studies (see, e.g.,[4]), our interest concerns only the vertical displacement. 

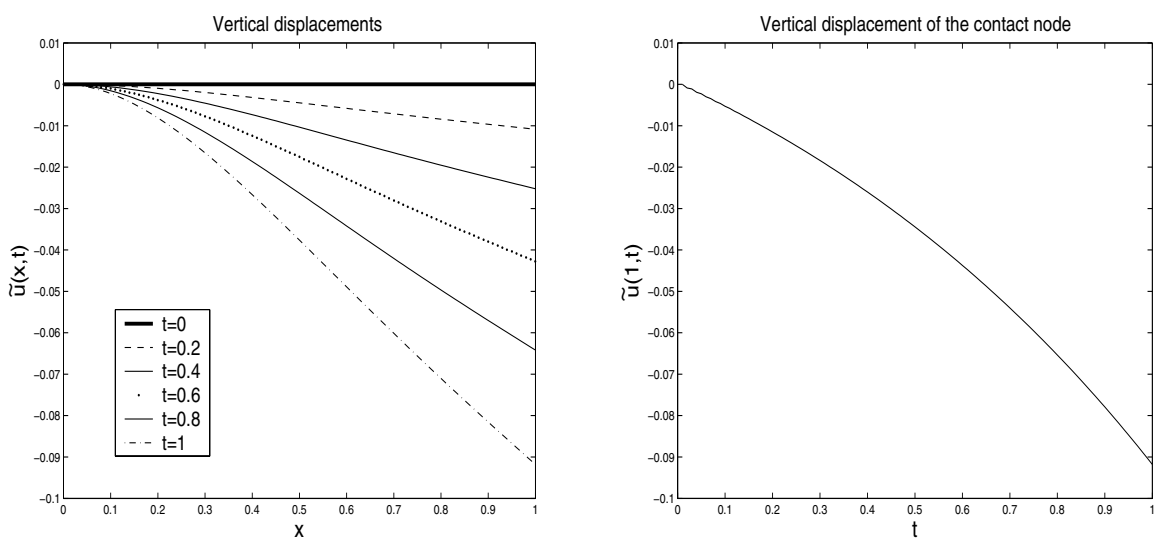

FigurE 4. Example 2: vertical displacements at different times and evolution in time of the vertical displacement of the contact node.
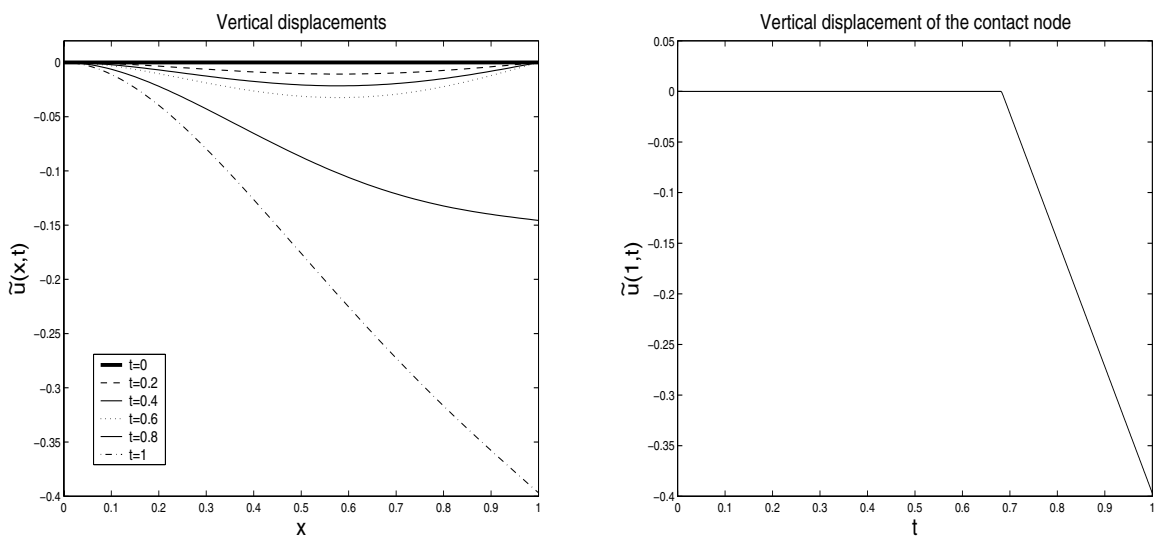

FiguRE 5. Example 3: vertical displacements at several times and evolution in time of the vertical displacement of the contact node.

The following data were used in the simulations:

$$
\begin{aligned}
& T=1 \mathrm{~s}, \quad a_{2}=1000 \mathrm{~N}, \quad c_{2}=1 \mathrm{~N} \cdot \mathrm{s}, \quad c_{V}=3000, \quad \mu=10^{-10}, \\
& \rho=10^{-4} \mathrm{~kg} / \mathrm{m}^{3}, \quad f_{V}(x, t)=-10000 \mathrm{t} \mathrm{N} / \mathrm{m} \text { in }[0,1], \\
& \tilde{u}_{0}(x)=0 \mathrm{~m}, \quad \tilde{v}_{0}(x)=0 \mathrm{~m} / \mathrm{s} \quad \text { in }[0,1] .
\end{aligned}
$$

Taking $h=k=10^{-3}$, in Figures 5 and 6 we show the numerical results obtained. In Figure 5 , the vertical displacements at several times (left) and the evolution in time of the vertical displacement of the contact node (right) are plotted. Moreover, the tangential stress is depicted in Figure 6. As we can observe, the contact node is clamped until time $t=0.68 \mathrm{~s}$, when the tangential stress $\sigma_{V}(1, t)$ reaches the friction bound.

\subsubsection{Fourth example: oscillating forces}

As a final test, we have considered a beam of length $L=1 \mathrm{~m}$ with its left end rigidly attached, and no gap is assumed between the right end and the obstacle $(g=0 \mathrm{~m})$. Coulomb's contact conditions were employed with 


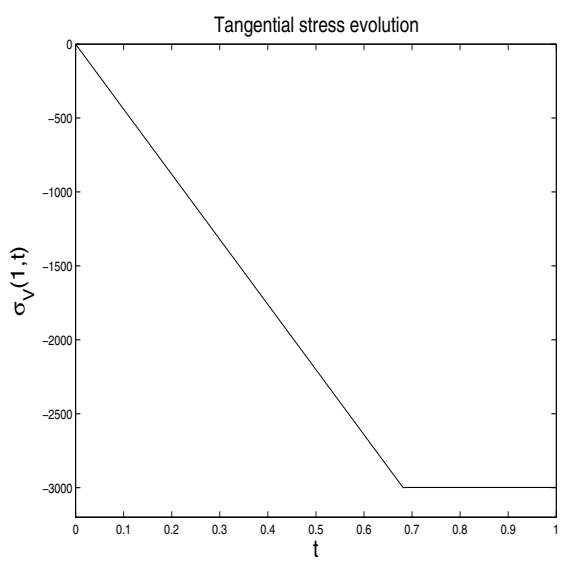

Figure 6. Example 3: evolution in time of the tangential stress.
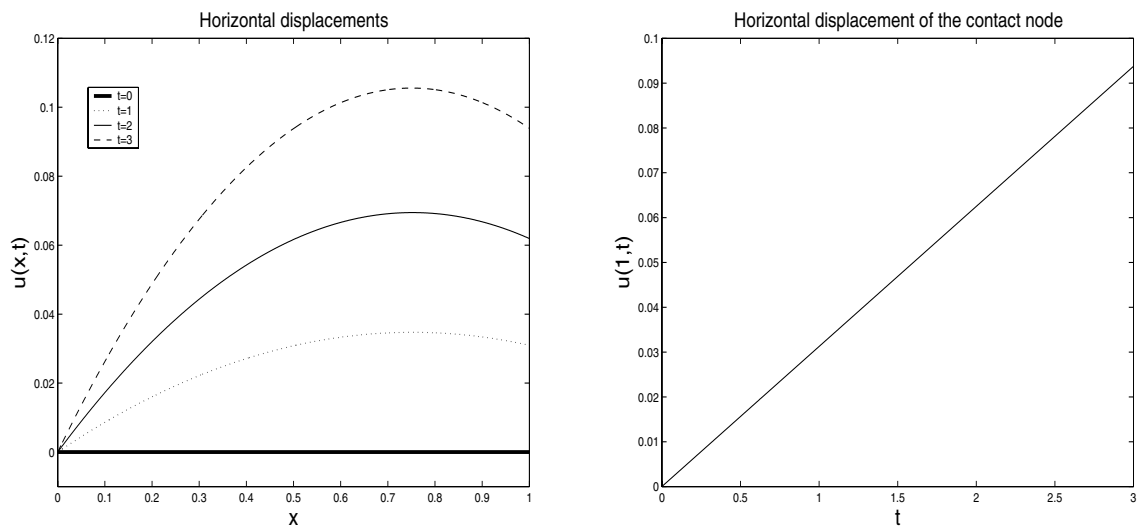

Figure 7. Example 4: horizontal displacements at different times and evolution in time of the horizontal displacement of the contact node.

the following data:

$$
\begin{aligned}
& T=3 \mathrm{~s}, \quad a_{1}=a_{2}=1000 \mathrm{~N}, \quad c_{1}=c_{2}=1 \mathrm{~N} \cdot \mathrm{s}, \\
& \rho=10^{-4} \mathrm{~kg} / \mathrm{m}^{3}, \quad c_{H}=10^{3}, \quad c_{V}=5 \times 10^{4}, \quad \mu=10^{-10}, \\
& f_{H}(x, t)=125 \mathrm{t} \mathrm{N} / \mathrm{m}, \quad f_{V}(x, t)=10000 \sin \frac{\pi t}{2} \mathrm{~N} / \mathrm{m} \text { in }[0,1], \\
& u_{0}(x)=\tilde{u}_{0}(x)=0 \mathrm{~m}, \quad v_{0}(x)=\tilde{v}_{0}(x)=0 \mathrm{~m} / \mathrm{s} \quad \text { in }[0,1] .
\end{aligned}
$$

Discretization parameters $h=k=0.001$ were used for solving the corresponding discrete problem $\mathrm{VP}^{h k}$. The horizontal displacements at several times and the evolution in time of the horizontal displacement of the contact node are shown in Figure 7. We notice that there is always penetration of the beam into the obstacle.

The vertical displacements at several times and the evolution in time of the vertical displacement of the contact node are plotted in Figure 8. Moreover, in Figure 9 it is shown the evolution in time of the friction bound $h(t)=c_{V} u(1, t)$ (left) and the tangential stress (right). Finally, the sum of $L^{2}$-norms of $u$ and $\tilde{u}$ is plotted in Figure 10 depending on the deformability and friction coefficients $c_{H}$ and $c_{V}$. The stability of the solution is clearly observed. 

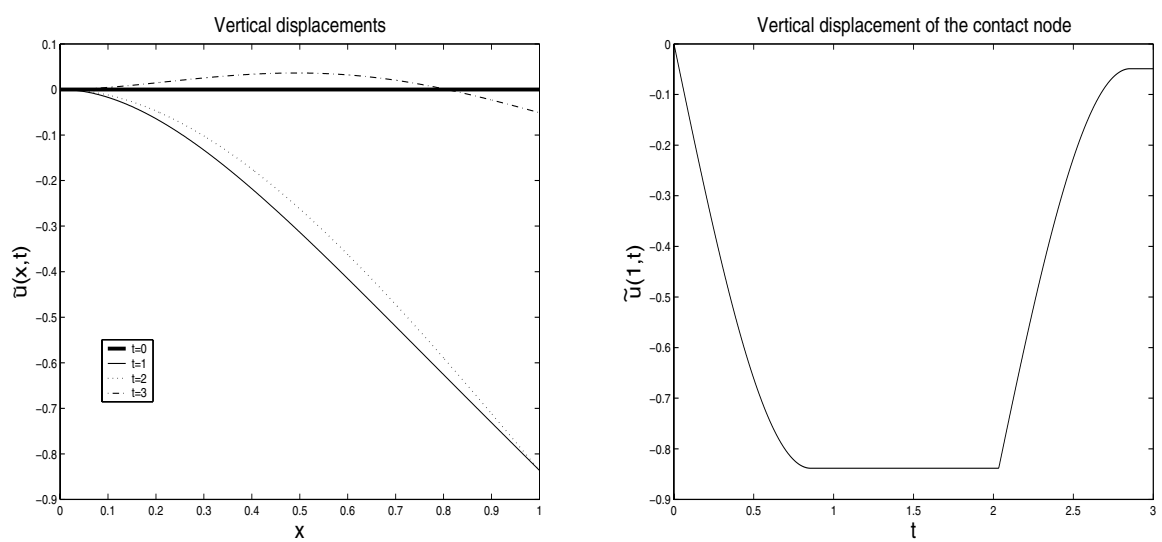

FiguRE 8. Example 4: vertical displacements at different times and evolution in time of the vertical displacement of the contact node.
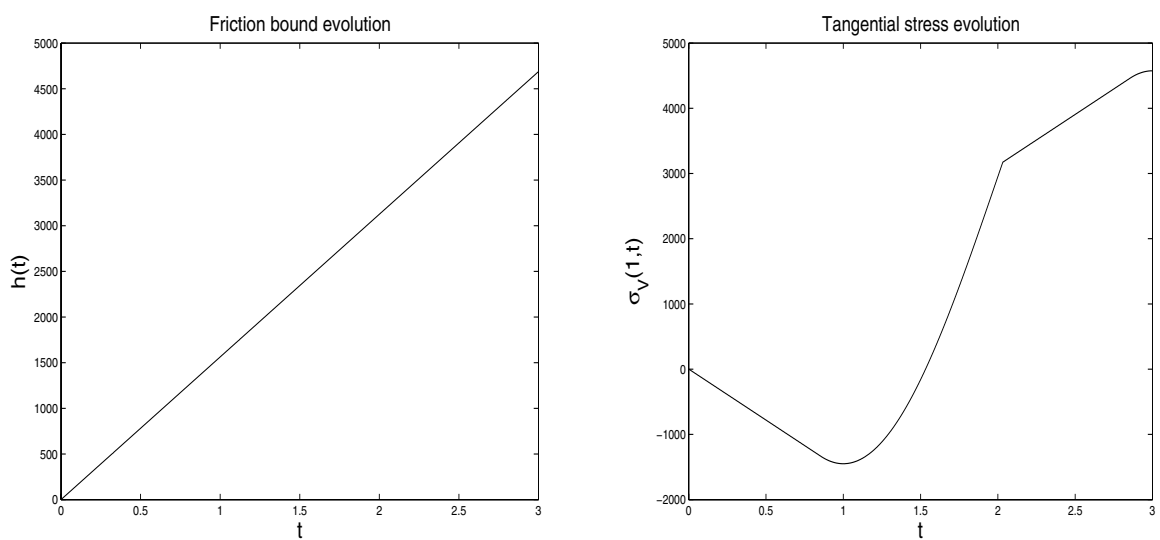

Figure 9. Example 4: evolution in time of the friction bound and the tangential stress.

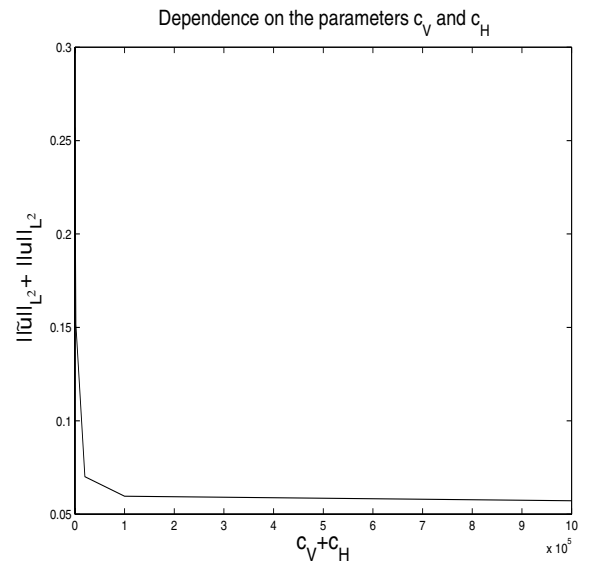

FiguRE 10. Example 4: sum of the $L^{2}$-norms depending on $c_{H}$ and $c_{V}$. 


\section{REFERENCES}

[1] K.T. Andrews, M. Shillor and S. Wright, On the dynamic vibrations of an elastic beam in frictional contact with a rigid obstacle. J. Elasticity 42 (1996) 1-30.

[2] K.T. Andrews, L. Chapman, J.R. Fernández, M. Fisackerly, M. Shillor, L. Vanerian and T. VanHouten, A membrane in adhesive contact. SIAM J. Appl. Math. 64 (2003) 152-169.

[3] K.T. Andrews, J.R. Fernández and M. Shillor, A thermoviscoelastic beam with a tip body. Comput. Mech. 33 (2004) $225-234$.

[4] K.T. Andrews, J.R. Fernández and M. Shillor, Numerical analysis of dynamic thermoviscoelastic contact with damage of a rod. IMA J. Appl. Math. 70 (2005) 768-795.

[5] A. Bermúdez and C. Moreno, Duality methods for solving variational inequalities. Comput. Math. Appl. 7 (1981) $43-58$.

[6] M. Campo, J.R. Fernández and J.M. Viaño, Numerical analysis and simulations of a quasistatic frictional contact problem with damage. J. Comput. Appl. Math. 192 (2006) 30-39.

[7] O. Chau, J.R. Fernández, W. Han and M. Sofonea, A frictionless contact problem for elastic-viscoplastic materials with normal compliance and damage. Comput. Methods Appl. Mech. Eng. 191 (2002) 5007-5026.

[8] X. Cheng and W. Han, Inexact Uzawa algorithms for variational inequalities of the second kind. Comput. Methods Appl. Mech. Eng. 192 (2003) 1451-1462.

[9] P.G. Ciarlet, The finite element method for elliptic problems, in Handbook of Numerical Analysis, P.G. Ciarlet and J.L. Lions Eds., Vol. II (1991) 17-352.

[10] G. Duvaut and J.L. Lions, Inequalities in mechanics and physics. Springer-Verlag, Berlin (1976).

[11] J.R. Fernández, M. Shillor and M. Sofonea, Numerical analysis and simulations of quasistatic frictional wear of a beam (submitted).

[12] A.C. Galucio, J.-F. Deü and R. Ohayon, Finite element formulation of viscoelastic sandwich beams using fractional derivative operators. Comput. Mech. 33 (2004) 282-291.

[13] R. Glowinski, Numerical methods for nonlinear variational problems. Springer, New York (1984).

[14] W. Han and M. Sofonea, Quasistatic contact problems in viscoelasticity and viscoplasticity. American Mathematical SocietyIntl. Press (2002).

[15] W. Han, K.L. Kuttler, M. Shillor and M. Sofonea, Elastic beam in adhesive contact. Int. J. Solids Struct. 39 (2002) 1145-1164.

[16] A. Klarbring, A. Mikelić and M. Shillor, Frictional contact problems with normal compliance. Int. J. Eng. Sci. 26 (1988) 811-832.

[17] K.L. Kuttler, A. Park, M. Shillor and W. Zhang, Unilateral dynamic contact of two beams. Math. Comput. Model. 34 (2001) 365-384.

[18] T.A. Laursen, Computational contact and impact mechanics: fundamentals of modeling interfacial phenomena in nonlinear finite element analysis. Springer, Berlin (2002).

[19] P.D. Panagiotopoulos, Inequality problems in mechanics and applications. Convex and nonconvex energy functions. Birkhäuser Boston, Boston (1985).

[20] I. Romero, The interpolation of rotations and its application to finite element models of geometrically exact rods. Comput. Mech. 34 (2004) 121-133.

[21] I. Romero and F. Armero, An objective finite element approximation of the kinematics of geometrically exact rods and its use in the formulation of an energy-momentum conserving scheme in dynamics. Int. J. Numer. Meth. Eng. 54 (2002) $1683-1716$.

[22] M. Sofonea, M. Shillor and R. Touzani, Quasistatic frictional contact and wear of a beam. Dyn. Contin. Discrete I. 8 (2000) 201-218.

[23] G.E. Stavroulakis and H. Antes, Nonlinear boundary equation approach for inequality 2-D elastodynamics. Eng. Anal. Bound. Elem. 23 (1999) 487-501.

[24] P. Wriggers, Computational contact mechanics. John Wiley and Sons Ltd (2002).

[25] H.W. Zhang, S.Y. He, X.S. Li and P. Wriggers, A new algorithm for numerical solution of 3D elastoplastic contact problems with orthotropic friction law. Comput. Mech. 34 (2004) 1-14. 